\title{
Nanocolumnar growth of thin films deposited at oblique angles: beyond the tangent rule
}

Running title: Nanocolumnar growth at oblique angles

Running Authors: Alvarez et al.

\author{
Rafael Alvarez ${ }^{\text {a) }}$, Carmen Lopez-Santos, Julian Parra-Barranco, Victor Rico and \\ Angel Barranco
}

Instituto de Ciencia de Materiales de Sevilla (CSIC-US), c/Americo Vespucio 49, 41092 Seville, Spain

\section{Jose Cotrino}

Instituto de Ciencia de Materiales de Sevilla (CSIC-US), c/Americo Vespucio 49, 41092 Seville, Spain and Departamento de Física Atómica, Molecular y Nuclear (Universidad de Sevilla). Av. Reina Mercedes, s/n, 41012 Seville, Spain

\section{Agustin R. Gonzalez-Elipe and Alberto Palmero}

Instituto de Ciencia de Materiales de Sevilla (CSIC-US), c/Americo Vespucio 49, 41092 Seville, Spain

\section{a) Electronic mail: rafael.alvarez@icmse.csic.es}

The growth of nanostructured physical vapor deposited thin films at oblique angles is becoming a hot topic for the development of a large variety of applications. Up to now, empirical relations, such as the so-called tangent rule, have been uncritically applied to account for the development of the nanostructure of these thin films even when they do not accurately reproduce most experimental results. In the present paper, the growth of thin films at oblique angles is analyzed under the premises of a recently proposed surface trapping mechanism. We demonstrate that this process mediates the effective shadowing area and determines the relation between the incident angle of the deposition flux and the 
tilt angle of the columnar thin film nanostructures. The analysis of experimental data for a large variety of materials obtained in our laboratory and taken from the literature supports the existence of a connection between the surface trapping efficiency and the metallic character of the deposited materials. The implications of these predictive conclusions for the development of new applications based on oblique angle deposited thin films are discussed.

\section{INTRODUCTION}

Physical vapor deposition (PVD) of thin films at oblique angles is known for being a reliable technique to synthesize nanostructured and sculptured thin films with anisotropic properties, such as dichroism, birefringence or anisotropic resistivity. ${ }^{1-3}$ This anisotropy combined with their high open porosity $^{4-7}$ are essential for other applications such as Bragg reflectors with tunable optical response, ${ }^{8}$ templates for nanocomposite films, ${ }^{9-12}$ broad band antireflection coatings, ${ }^{13,14}$ optical microresonators, ${ }^{15}$ light emitting diodes, ${ }^{16}$ photovoltaic cells, ${ }^{17}$ advanced plasmon photocatalysis, ${ }^{18,19}$ microfluidic sensors, ${ }^{20}$ transparent conductive electrodes ${ }^{21}$ and many others. In this technique, a given material is sublimated in a vacuum reactor, either thermally or assisted by an electron beam, yielding vapor species that follow straight trajectories in a "line of sight" configuration with respect to the substrate, and giving rise to thin films with well-defined tilted nanocolumnar structures. Indeed, these structures represent a key feature for the implementation of other applications linked to well-defined intercolumnar spaces in the film, thus making it suitable as a host or template for further material manufacturing. In reference 20 for instance, we have recently demonstrated that the relative orientation of 
nanocolumns in stacks of $\mathrm{TiO}_{2} / \mathrm{SiO}_{2}$ layers directly determined the efficiency of the material as a Bragg reflector. Also, in reference 22 it is proven that the inherent structural anisotropy of the tilted array of nanocolumns induces a direction-dependent electrical bistability when embedded in a polymer matrix, thus opening numerous possibilities regarding anisotropic applications. Furthermore, in reference 23 it is shown that the tilt angle of the columnar structures had a tremendous influence in the total surface area of the layers, indicating that any specific application that requires maximum specific area, e.g., for light or molecule surface collection, is strongly mediated by the tilt angle of these structures.

Due to the relevance of this issue, much work has been devoted in the literature to understand the connection between the angle aligning the evaporation source and the growing film, $\alpha$, and the tilt angle of the columns, $\beta$, a relation of great importance to foresee the development of the abovementioned anisotropic nanocolumnar structure and, hence, the film properties. ${ }^{24-28}$ Yet, the formulas proposed to link $\alpha$ and $\beta$ are known to provide first estimations, but fail when systematically applied to actual thin films prepared by PVD at oblique angles. For instance, the so-called tangent rule

$$
\tan \alpha=2 \tan \beta
$$

is known for providing good estimations of $\beta$ when $\alpha$ is kept below $60^{\circ}$, but fails for higher incident angles. ${ }^{24}$ Moreover, by employing a purely ballistic model, Tait et al. ${ }^{25}$ proposed the so-called cosine rule,

$$
\beta=\alpha-\arcsin \left(\frac{1-\cos \alpha}{2}\right)
$$


that yields good results for some materials but fails for others. An implicit assumption in these rules is that the relation between $\alpha$ and $\beta$ is purely geometrical and, thus, independent of the nature of the deposited material, a hypothesis that can be considered doubtful for most conditions. ${ }^{29}$

In reference 30 , following similar philosophy than in previous works, ${ }^{31-34}$ we developed a Monte Carlo ballistic model to describe the PVD deposition of thin films at oblique angles and at low temperatures. In that work, we proposed that short-range interactions between vapor species and the film surface could influence the film growth through a so-called surface trapping mechanism. In contrast with classical ballistic models, where the deposition species follow straight trajectories up to their impingement on the film, this mechanism considers that vapor species moving at distances from the surface below 3-4 angstroms may modify their trajectory under the action of short-range (e.g., electrostatic and/or van der Waals) interactions. ${ }^{35}$ The assumption of a trapping mechanism in the growth model yielded a remarkable agreement between experimental and simulated nanostructures of $\mathrm{TiO}_{2}$ thin films, as well as an accurate prediction of the relation between $\alpha$ and $\beta$ for this material. In this paper we aim at proving the general occurrence of this mechanism in a broad range of PVD conditions at oblique angles for numerous materials: keeping this purpose in mind, we have analyzed numerous experimental data obtained in our laboratory and taken from the literature. It has been concluded that, in most cases, the widely and uncritically utilized empirical rules (i.e., tangent and cosine rules) are unable to properly explain the dependence of the columnar growth on the deposition system and the evaporated material, and that concepts such as angular broadening of the vapor flux and/or the surface trapping efficiency are required 
to properly describe the morphological evolution of the films during growth, as well as to tailor their nanostructure for final applications.

\section{METHODOLOGY}

\section{A. Experimental method}

$\mathrm{SiO}_{2}, \mathrm{Ta}_{2} \mathrm{O}_{5}$, ITO (indium tin oxide) and metallic Ti thin films were deposited by electron beam-assisted PVD at different oblique angles, $\alpha=60^{\circ}, 70^{\circ}, 80^{\circ}$ and $85^{\circ}$. Films were deposited on silicon substrates using the same electron beam evaporation set-up (see Figure 1). To ensure the total oxidation of the oxide thin films, evaporations were carried out in an $\mathrm{O}_{2}$ atmosphere at a pressure of $10^{-2} \mathrm{~Pa}$ using $\mathrm{SiO}_{2}, \mathrm{Ta}_{2} \mathrm{O}_{5}$ and ITO pellets as precursor materials, whereas the metallic Ti thin films were prepared using Ti pellets in the absence of $\mathrm{O}_{2}$ in the reactor. In all cases, the background pressures were low enough to neglect collisions in the gaseous phase during the flight of the evaporated species from the crucible to the substrate, situated at a distance of $50 \mathrm{~cm}$. The cross-sectional images of the films were taken with a field emission scanning electron microscope (FESEM), HITACHI S5200, cleaving the silicon substrates.

\section{B. Simulation}

The growth model used is described in detail in reference 30. Vapor particles arriving at the substrate are considered to follow a narrow Gaussian angular distribution function with nominal angle $\alpha$ and variance $\sigma$. Thus, it is assumed that they arrive at the film with an average angle defined by $\alpha$, being $\sigma$ a measure of the possible angular broadening of the vapor flux associated to the finite size of the source and/or the system geometry. The model also assumes that vapor species follow straight trajectories until 
they hit the surface, where they stick with probability 1 . However, when they move 3-4 angstroms away from the surface, it is considered that these may bend their trajectory due to short-range interactions with the film surface and stick at that location with probability $s_{t}$, the so-called surface trapping probability. Consequently, in this description, the value of $\sigma$ is determined by the geometry and size of the deposition set-up, regardless of the chemical nature of the evaporated material, whereas $s_{t}$ should only depend upon the nature of the interaction between evaporated species and the film surface. The physical mechanisms associated to these two parameters are discussed in more detail in reference 30.

The film growth is considered to take place in the so-called Zone I of the Structure Zone Model (i.e., $T_{f} / T_{m}<0.3$, where $T_{f}$ is the film temperature and $T_{m}$ the melting temperature of the deposited material), ${ }^{36}$ where surface shadowing mechanisms are known to dominate over thermally activated mobility processes. A MATLAB-based computer code, STRONG (Surface Trapping in Oblique Nanostructured Growths), has been developed to solve the presented model. An executable user-friendly version of this software can be freely downloaded from our webpage. ${ }^{37}$

\section{RESULTS AND DISCUSSION}

Figure 2 a shows the relationships between $\alpha$ and $\beta$ determined by the model, as a function of $s_{t}$ and $\sigma$, as well as the trends derived by the tangent and cosine rules, eqs.(1-2), for $\alpha \geq 40^{\circ}$. The curves for $s_{t} \geq 0.1$ are little sensitive to the particular value of $\sigma$ : in figure $2 \mathrm{a}$ we have plotted them for $\sigma=6^{\circ}$, whereas in figure $2 \mathrm{~b}$ for $\sigma=0^{\circ}$. By 
contrast, since the curve for $s_{t}=0$ is highly dependent on $\sigma$, we have plotted the two cases, $\sigma=0^{\circ}$ and $\sigma=6^{\circ}$ in both figures. The comparison between these two figures illustrate the abovementioned weak dependence on the value of $\sigma$ whenever $s_{t} \geq 0.1$, with a maximum variation around $2^{\circ}$ in all these cases. Moreover, as a general trend, we find that the higher the value of $s_{t}$ is, the less tilted the nanocolumns are. This indicates that surface trapping processes are modifying the geometrical shadowing process and that the columnar growth must be described by means of an effective shadowing area (see Figure 3). With this term we denote the region around the thin film surface features in which vapor species are trapped, thus casting an additional shadow on the film surface. Interestingly, the results of the model follow the tangent rule, eq.(1) when $\alpha$ is lower than $\sim 60^{\circ}$. For these low evaporation angles, the curve with $s_{t}=0$ and $\sigma=0^{\circ}$ falls on top of that described by the tangent rule, whereas the curves with $s_{t}>0$ and $\sigma>0^{\circ}$ follow same trend but fall just below it in this range. This demonstrates that the influence of the surface trapping mechanism is increasingly relevant for higher incident angles, and that it is coherent with the physical nature of the interaction: trapping processes are more efficient whenever vapor particles fly over the film surface for longer times, i.e., when they approach the film at glancing trajectories.

In Figure 4 we show the obtained cross-sectional FESEM images of the deposited films. A first assessment of their nanostructures evidences different columnar growths and tilt angles in each case, a result proving that the relation between $\alpha$ and $\beta$ must be material-dependent and, therefore, not accountable by a simple universal equation that only considers geometrical relationships. In Figure 2a, the values of $\beta$ have been plotted 
as a function of $\alpha$ for each film, together with the already reported results on $\mathrm{TiO}_{2}$ deposited in the same experimental setup and similar conditions. ${ }^{30}$ Overall, the data in Figure 2a show a good agreement between experiments and calculations regarding the range of experimental values of $\beta$. Furthermore, there is a remarkable concordance between the values of $\beta$ for a given material and the curve obtained for a constant value of $s_{t}$ : this strongly supports our hypothesis that $s_{t}$ only depends on the chemical nature of the sublimated material and of the composition of the film. The lack of accuracy of eqs.(1-2) is also apparent in Figure 2a.

The data in Figure 2a also support the existence of a certain link between the nanostructures of the different materials based on their chemical nature. Results for $\mathrm{TiO}_{2}$ match those in reference 30 where it was found that the best values describing the growth were $s_{t}=0.12$ and $\sigma=6^{\circ}$. For Ti thin films, on the other hand, we have found that they are well-described with the pair of values $s_{t} \sim 0$ and $\sigma=6^{\circ}$. The common value $\sigma=6^{\circ}$ agrees with our hypothesis that $\sigma$ should only depend on the geometrical features of the deposition setup. For $\mathrm{SiO}_{2}, \mathrm{Ta}_{2} \mathrm{O}_{5}$ and ITO films the calculation of $\sigma$ is not straightforward due to the weak dependence of $\sigma$ on $\beta$ when $s_{t} \geq 0.1$. Conversely, the different $s_{t}$ values for $\mathrm{Ti}$ and $\mathrm{TiO}_{2}$ agree with the idea that, in comparison with the $\mathrm{Ti}-\mathrm{Ti}$ bond structure of the former, the $\mathrm{O}$ - $\mathrm{Ti}$ bond structures in the $\mathrm{TiO}_{2}$ film surface modify the interaction potential with the sublimated species. In Figure $2 a$, it is also apparent that the values of $\beta$ for other oxide thin films, like $\mathrm{SiO}_{2}$ and $\mathrm{Ta}_{2} \mathrm{O}_{5}$, fall along the $s_{t}=1$ curve. Unfortunately, the different behaviour of these oxide materials indicates that predicting the actual value of $s_{t}$ for a film with a certain composition is not straightforward at 
present. An additional difficulty in this regard is that, for many materials, sublimation produces more than one chemical species which, most likely, would interact differently with the film surface. In these cases, the trapping probability should be understood as an average over each species' trapping probability. For illustration purposes, in Figure 5 we have included cross-sectional images of the simulated film nanostructures for $\alpha=80^{\circ}$, $\sigma=6^{\circ}$ and $s_{t}=0(\mathrm{Ti}), s_{t}=0.1(\mathrm{Si}), s_{t}=0.3(\mathrm{ITO})$ and $s_{t}=1\left(\mathrm{SiO}_{2}\right)$, together with the FESEM images of $\mathrm{Ti}, \mathrm{TiO}_{2}, \mathrm{ITO}$ and $\mathrm{SiO}_{2}$, respectively. The comparison of the experimental and calculated nanostructures sustains that the model reproduces fairly well the growth of all the studied materials.

To further assess the validity of the trapping mechanism, in Figure $2 b$ we have plotted additional data for $\mathrm{TiO}_{2}, \mathrm{Si}, \mathrm{Ni}$ and $\mathrm{Ag}$ taken from reference 29, together with the results of the model. Among the many experimental data in the literature on this subject, we have chosen this set because they correspond to films grown at room temperature, in the same experimental setup and under similar conditions, features that permit a direct comparison with our theory and experimental data. $\mathrm{For} \mathrm{TiO}_{2}$, the $\beta$ values agree with those obtained in our laboratory, both matching with the curve defined by $s_{t} \sim 0.1$, and confirm that $s_{t}$ only depends on the nature of sublimated and deposited materials. The same curve, $s_{t} \sim 0.1$, also reproduces the deposition of $\mathrm{Si}$, which differs from that of $\mathrm{SiO}_{2}$ thin films, with $s_{t} \sim 1$, sustaining again that the presence of metal-oxygen bonding structures at the surface lattice and/or the different nature of the deposition species change the intensity of the interaction and, consequently, the surface trapping probability. $\mathrm{Ni}$ and Ag films follow the curve defined by $s_{t} \sim 0$ and $\sigma \sim 0^{\circ}$, and support the 
assumption that $\sigma$ basically depends on the geometrical features of the experimental setup. It must be noted that any possible influence of $\sigma$ is not apparent for the $\mathrm{TiO}_{2}$ and Si cases because they follow the curve $s_{t} \sim 0.1$ which is little affected by the value of the former parameter. In Figure 2a-b, we also find a remarkable result: all metallic elemental thin films (Ti, Ni and $\mathrm{Ag}$ ) are well described with $s_{t} \sim 0$, whereas elemental Si thin films, with covalent character, are well described with $s_{t} \sim 0.1$. Interestingly, compound thin films (ITO, $\mathrm{SiO}_{2}, \mathrm{TiO}_{2}$ and $\mathrm{Ta}_{2} \mathrm{O}_{5}$ ) are all characterized by higher values of this parameter $\left(0.1 \leq s_{t} \leq 1\right)$.

Actual values of $s_{t}$ for specific materials are not available at this moment due to the lack of first principles calculations or experimental surface trapping data in the literature. This brings up the question of whether $s_{t}$ is actually representing a physical phenomenon or it is just a mere fitting parameter within a growth model with no further implications. Regarding the results above, we have shown that all experimental values of $\beta$ for each material follow a single theoretical curve defined by a constant value of $s_{t}$. Moreover, a given material deposited in different experimental setups (e.g., the two sets of $\mathrm{TiO}_{2}$ films in Figure 2) is described by a single curve corresponding to an unique value of $s_{t}$, whereas different materials deposited in the same experimental setup are well described by a single value of $\sigma$ (this effect is noticeable by comparing $\mathrm{TiO}_{2}$ and $\mathrm{Ti}$ produced in our laboratory and $\mathrm{Ag}$ and $\mathrm{Ni}$ from reference 29). We would also like to stress that for $\alpha<60^{\circ}$, the solutions of our model follow the curve defined by the tangent rule, eq.(1), which is known to be valid for low values of $\alpha,{ }^{24}$ a fact that further supports the validity of the model. These evidences strongly suggest that the surface trapping 
mechanism is actually a relevant process that strongly influences the film nanostructuration and the columnar growth in PVD of thin films at oblique angles. Finally, we would like to remark that in this paper we have not analyzed the effect of thermally-induced mobility processes. As mentioned above, our model assumes a Zone I growth of the SZM purely governed by surface shadowing effects, so the analysis is only valid for films grown at low temperatures. Although some thermal processes should be present in the conditions analyzed in this paper, the low values of $T_{f} / T_{m}$ in all studied cases and the good match between experimental and simulated microstructures in Figures $2 a, 2 b$ and 5 ensure that thermal diffusion effects are not noticeable in our conditions.

Overall, our model accounts for the material-dependent features by means of an effective shadowing area, mediated by surface trapping processes. The fact that no adparticles diffusion processes are considered in Zone I of the SZM, implies that experimental quantities such as the evaporation (deposition) rate of material, have no or little influence on the results. Furthermore, our analysis has been performed in typical ebeam assisted PVD conditions, i.e., when the gas pressure in the reactor is low enough to make negligible the number of collisions of evaporated species from the source to the film. In other conditions, an increase of the background pressure would result in higher values of $\sigma$, and hence in the growth of well separated columns with larger diameters and rounder tips. ${ }^{30}$ Finally, our model assumes an amorphous structure of the materials and thus no potential barriers, preferential directions or textures are considered.

In this paper, we have developed a sound framework that describes the PVD of thin films at oblique angles and at low temperatures. We have shown that the surface trapping and the angular broadening of the vapor flux are relevant mechanisms that 
accurately explain the intrinsic relation between $\alpha$ and $\beta$ for different materials and deposition reactors, clearly improving the accuracy of the tangent or the cosine rules (Eqs. 1-2). As mentioned in the introduction, the tilt angle of the columnar structures is a key morphological property influencing critical aspects of the film such as the open porosity or its anisotropic properties, for instance. In this regard, the results presented in this paper are of relevance, not only from a fundamental point of view, but also concerning the tailoring and fabrication of nanostructured films with optimized morphologies for functional applications.

\section{CONCLUSIONS}

Going beyond the common growing process schemes in the literature, in this paper we have rationalized the formation of tilted nanocolumns in evaporated thin films prepared at glancing angles. This geometrical arrangement, acknowledged as the main characteristic feature of thin films prepared in an oblique configuration, is of the outmost importance for most applications relying on this type of thin film microstructure. The tilting angles of the nanocolumns for a large set of thin films deposited by PVD at oblique angles and low temperatures have been accounted for by means of surface trapping mechanisms. We have found that these mechanisms affect the effective area responsible for the surface shadowing of vapor species and explain the different relations between the incident angle of the deposition flux and the tilt angle of the growing columns. Furthermore, we have obtained that the complexity associated to the system geometry and the material-dependent growth can be simplified through two fundamental parameters: i) the angular broadening of the incident vapor flux, and ii) the surface 
trapping probability. As a result, it seems that the surface trapping efficiency is the lowest for metal thin films. The proposed mechanism provides a new framework to classify and analyze the growth of films by PVD at low temperatures and oblique angles which, going beyond the heuristic tangent and cosine rules, provides a framework to tailor the microstructure of oblique deposited thin films. This rationalization of the nanocolumnar growth during oblique angle deposition of thin films has direct implications for the development of materials with well controlled microstructure where the tilting angle and geometry of the nanocolumns play a critical role.

\section{ACKNOWLEDGMENTS}

We thank the Junta de Andalucía (Projects P09-CTS- 5189, TEP5283 and FQM-6900) and the Ministry of Science and Innovation (Projects CONSOLIDER CSD2008-00023, MAT2010-21228, MAT2010-18447) for financial support.

${ }^{1}$ A. Lakhtakia, M. W. McCall, J. A. Sherwin, Q. H. Wu, and I. J. Hodgkinson, Opt. Commun. 194, 33 (2001).

${ }^{2}$ G. Mbise, G. B. Smith, G. A. Niklasson, and C. G. Granqvist, Appl. Phys. Lett. 54, 987 (1989).

${ }^{3}$ I. Hodgkinson and Q. H. Wu, Appl. Optics 38, 3621 (1999).

${ }^{4}$ D. J. Poxson, F. W. Mont, M. F. Schubert, J. K. Kim, and E. F. Schubert, Appl. Phys. Lett. 93, 101914 (2008).

${ }^{5}$ L. Gonzalez-Garcia, J. Parra-Barranco, J. R. Sanchez-Valencia, A. Barranco, A. Borras, A. R. Gonzalez-Elipe, M.C. Garcia-Gutierrez, J. J. Hernandez, D. R. Rueda, and T. A. Ezquerra, Nanotechnology 23, 205701 (2012). 
${ }^{6}$ Y. P. He, Z. Y. Zhang, and Y. P. Zhao, J. Vac. Sci. Technol., B 26, 1350 (2008).

${ }^{7}$ C. Buzea, K. Kaminska, G. Beydaghyan, T. Brown, C. Elliott, C. Dean, K. Robbie, J. Vac. Sci. Technol., B 23, 2545 (2005).

${ }^{8}$ J. J. Steele, A. C. van Popta, M. M. Hawkeye, J. C. Sit, and M. J. Brett, Sensor. Actuat. B-Chem. 120, 213 (2006).

${ }^{9}$ H.-Y. Yang, M.-F. Lee, C.-H. Huang, Y.-S. Lo, Y.-J. Chen, and M.-S. Wong, Thin Solid Films 518, 1590 (2009).

${ }^{10}$ G. K. Kiema, M. O. Jensen, and M. J. Brett, Chem. Mater. 17, 4046 (2005).

${ }^{11}$ J. R. Sanchez-Valencia, J. Toudert, A. Borras, A. Barranco, R. Lahoz, G. F. de la Fuente, F. Frutos, and A. R. Gonzalez-Elipe, Adv. Mater. 23, 848 (2011).

${ }^{12}$ L. Gonzalez-Garcia, I. Gonzalez-Valls, M. Lira-Cantu, A. Barranco, and A. R. Gonzalez-Elipe, Energy Environ. Sci. 4, 3426 (2011).

${ }^{13}$ J. A. Dobrowolski, D. Poitras, P. Ma, H. Vakil, and M. Acree, Appl. Optics 41, 3075 (2002).

${ }^{14}$ D. Poitras and J. A. Dobrowolski, Appl. Optics 43, 1286 (2004).

${ }^{15}$ Q. F. Xu, V. R. Almeida, R. R. Panepucci, and M. Lipson, Opt. Lett. 29, 1626 (2004).

${ }^{16}$ J. K. Kim, T. Gessmann, E. F. Schubert, J. Q. Xi, H. Luo, J. Cho, C. Sone, and Y. Park, Appl. Phys. Lett. 88, 013501 (2006).

${ }^{17}$ L. Gonzalez-Garcia, J. Idigoras, A. R. Gonzalez-Elipe, A. Barranco, and J. A. Anta, J. Photoch. Photobio., A, 241, 58 (2012).

${ }^{18}$ Y. He, P. Basnet, S. E. H. Murph, and Y. Zhao, ACS Appl. Mater. Interfaces 5, 11818 (2013).

${ }^{19}$ P. Basnet, G. K. Larsen, R. P. Jadeja, Y.-C. Hung, and Y. Zhao, ACS Appl. Mater. Interfaces 5, 2085 (2013).

${ }^{20}$ M. Oliva-Ramirez, L. Gonzalez-Garcia, J. Parra-Barranco, F. Yubero, A. Barranco, and A. R. Gonzalez-Elipe, ACS Appl. Mater. Interfaces 5, 6743 (2013). 
${ }^{21}$ A. L. Beaudry, R. T. Tucker, J. M. LaForge, M. T. Taschuk, M. J. Brett, Nanotechnology 23, 105608 (2012).

${ }^{22}$ P. P. Pillai, K. Paclawski, J. Kim, and B. A. Grzybowski, Adv. Mater. 25, 1623 (2013).

${ }^{23}$ K. M. Krause, M. T. Taschuk, K. D. Harris, D. A. Rider, N. G. Wakefield, J. C. Sit, J. M. Buriak, M. Thommes, and M. J. Brett, Langmuir 26, 4368 (2010).

${ }^{24}$ J. M. Nieuwenhuizen and H. B. Haanstra, Philips Tech. Rev. 27, 87 (1966).

${ }^{25}$ R. N. Tait, T. Smy, and M. J. Brett, Thin Solid Films 226, 196 (1993).

${ }^{26}$ I. Hodgkinson, Q. H. Wu, and J. Hazel, Appl. Optics 37, 2653 (1998).

${ }^{27}$ Q. Zhou, Z. Li, J. Ni, and Z. Zhang, Mater. Trans. 52, 469 (2011).

${ }^{28}$ B. Tanto, G. Ten Eyck, and T. M. Lu, J. Appl. Phys. 108, 026107 (2010).

${ }^{29}$ H. Zhu, W. Cao, G. K. Larsen, R. Toole, and Y. Zhao, J. Vac. Sci. Technol., B 30, 030606 (2012).

${ }^{30}$ R. Alvarez, L. Gonzalez-Garcia, P. Romero-Gomez, V. Rico, J. Cotrino, A. R. Gonzalez-Elipe, and A. Palmero, J. Phys. D Appl. Phys. 44, 385302 (2011).

${ }^{31}$ J. M. Garcia-Martin, R. Alvarez, P. Romero-Gomez, A. Cebollada, and A. Palmero, Appl. Phys. Lett. 97, 173103 (2010).

${ }^{32}$ R. Alvarez, P. Romero-Gomez, J. Gil-Rostra, J. Cotrino, F. Yubero, A. Palmero, and A. R. Gonzalez-Elipe, J. Appl. Phys. 108, 064316 (2010).

${ }^{33}$ R. Alvarez, J. M. Garcia-Martin, M. Macias-Montero, L. Gonzalez-Garcia, J. C. Gonzalez, V. Rico, J. Perlich, J. Cotrino, A. R. Gonzalez-Elipe, and A. Palmero, Nanotechnology 24, 045604 (2013).

${ }^{34}$ R. Alvarez, A. Palmero, L. O. Prieto-Lopez, F. Yubero, J. Cotrino, W. de la Cruz, H. Rudolph, F. H. P. M. Habraken, and A. R. Gonzalez-Elipe, J. Appl. Phys. 107, 054311 (2010).

${ }^{35}$ S. Muller-Pfeiffer, H. van Kranenburg, and J. C. Lodder, Thin Solid Films 213, 143 (1992). 
${ }^{36}$ I. Petrov, P. B. Barna, L. Hultman, and J. E. Greene, J. Vac. Sci. Technol., A 21, S117 (2003).

${ }^{37}$ The software STRONG is available at http://nanoscops.icmse.csic.es/

\section{Figure Captions}

Figure 1. Experimental set-up.

Figure 2. (Color online) Column tilt angle as a function of $\alpha$ for different values of the surface trapping probability, $s_{t}$, as obtained by the model. The curves corresponding to the tangent and the cosine rules, eq.(1-2), are also included. a) Calculations for $s_{t} \geq 0.1$ were carried out for $\sigma=6^{\circ}$. Symbols correspond to experimental results for $\mathrm{TiO}_{2},{ }^{30} \mathrm{SiO}_{2}$, $\mathrm{Ta}_{2} \mathrm{O}_{5}$, ITO, and metallic Ti deposited in our laboratory. b) Calculations for $s_{t} \geq 0.1$ were carried out for $\sigma=0^{\circ}$. Symbols correspond to experimental data from the literature ${ }^{29}$ for $\mathrm{TiO}_{2}, \mathrm{Si}, \mathrm{Ni}$ and $\mathrm{Ag}$.

Figure 3. (Color online) Scheme of the surface trapping process: in a classical ballistic approach, deposition species do not interact with the film surface and follow a straight trajectory until they land on the surface. In a more realistic approach, deposition species 
may get trapped due to short-range interactions with the surface, bend its trajectory and land at a different location.

Figure 4. (Color online) Cross-sectional FESEM images of the different thin films deposited in our laboratory for different values of $\alpha$. Solid lines illustrate the measured tilt angle of the columnar nanostructure (the value of $\beta$ is shown in the top right corner of each image).

Figure 5. Cross-sectional images of the simulated film nanostructures for $\alpha=80^{\circ}, \sigma=6^{\circ}$ and $s_{t}=0, s_{t}=0.1, s_{t}=0.3$ and $s_{t}=1$, which, according to Figure 2, describe the growth of $\mathrm{Ti}, \mathrm{Si}$, ITO and both $\mathrm{Ta}_{2} \mathrm{O}_{5}$ and $\mathrm{SiO}_{2}$ thin films, respectively. On the right side the corresponding FESEM images are included for comparison purposes. 


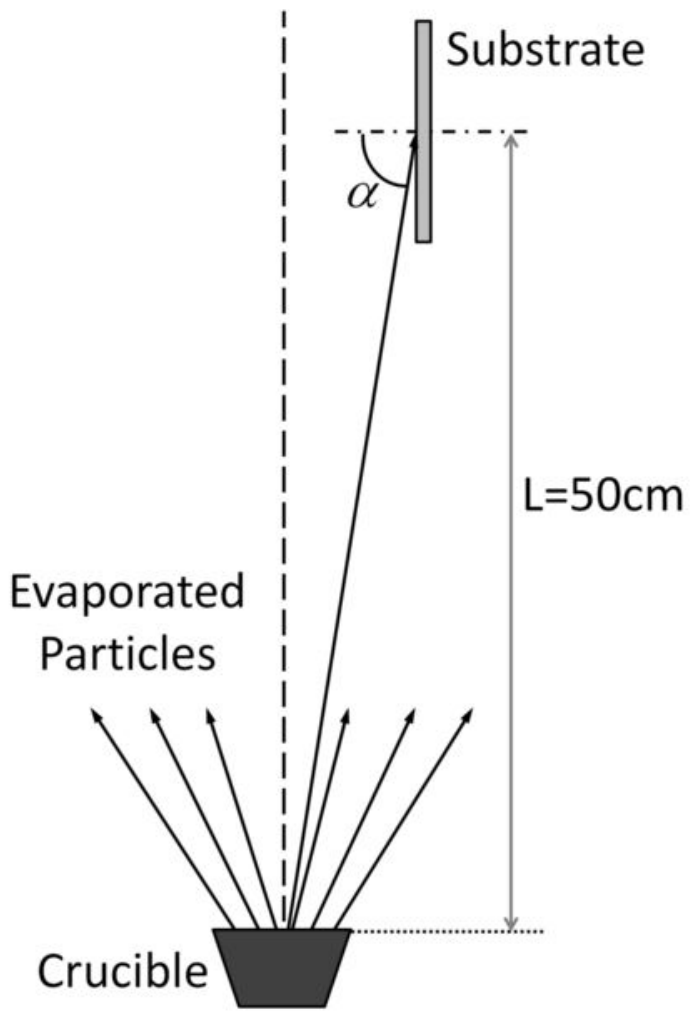




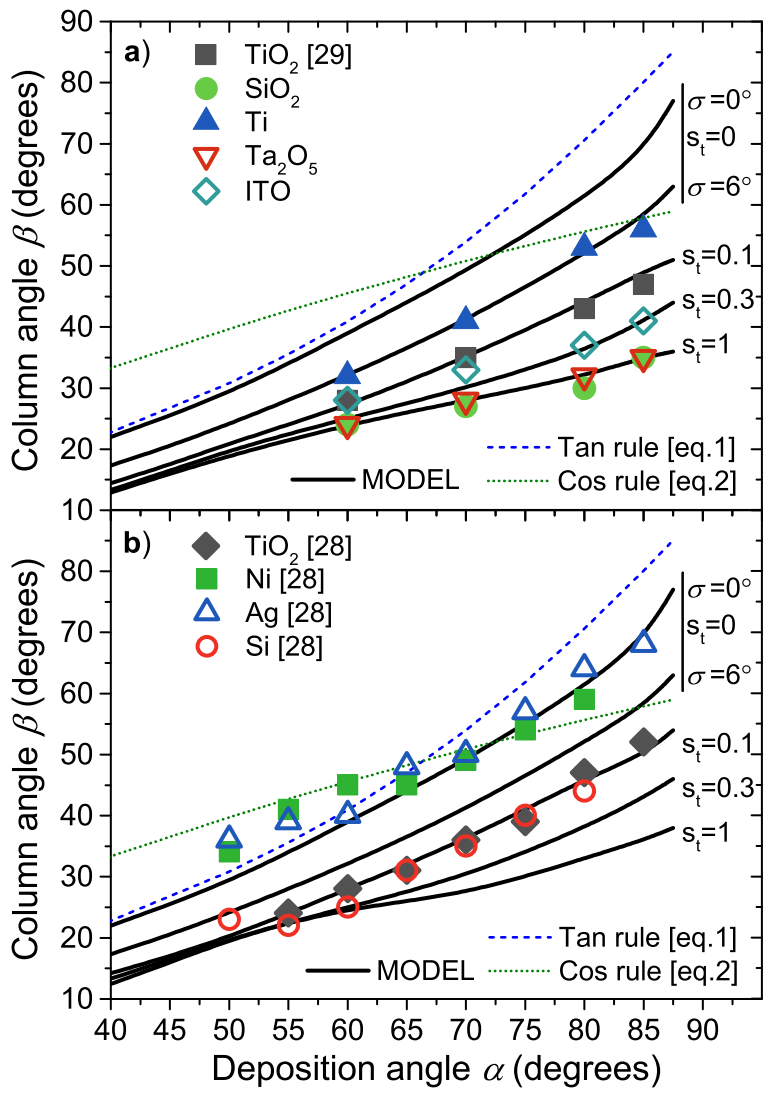




\section{Surface Trapping}

Mechanism 
$\mathrm{Ta}_{2} \mathrm{O}_{5}$

$\mathrm{SiO}_{2}$

ITO

$\mathrm{Ti}$

$\alpha=60^{\circ}$

$\alpha=70^{\circ}$

$\alpha=80^{\circ}$

$\alpha=85^{\circ}$
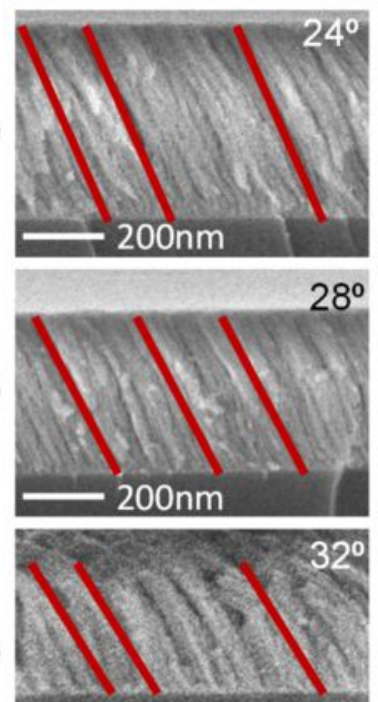

$200 \mathrm{~nm}$

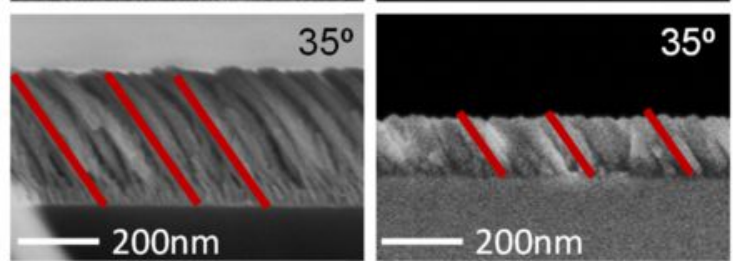

$100 \mathrm{~nm}$
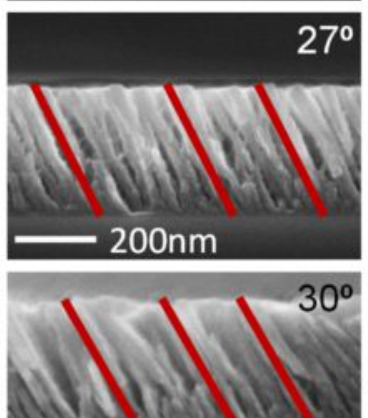

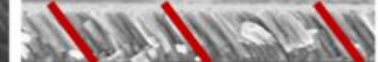

$200 \mathrm{~nm}$

$200 \mathrm{~nm}$

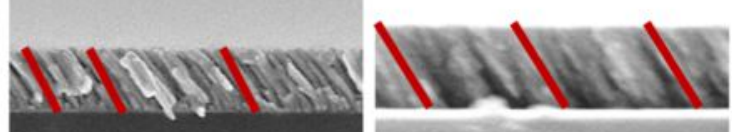

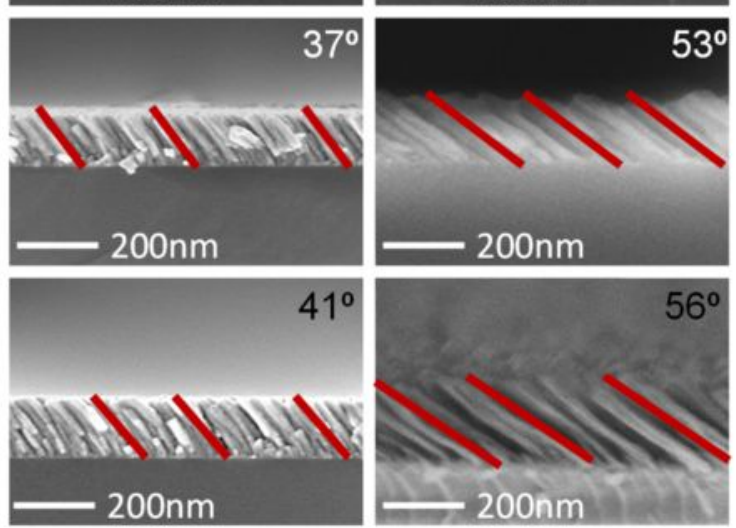

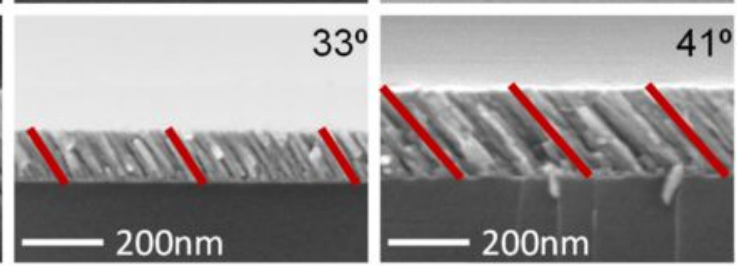



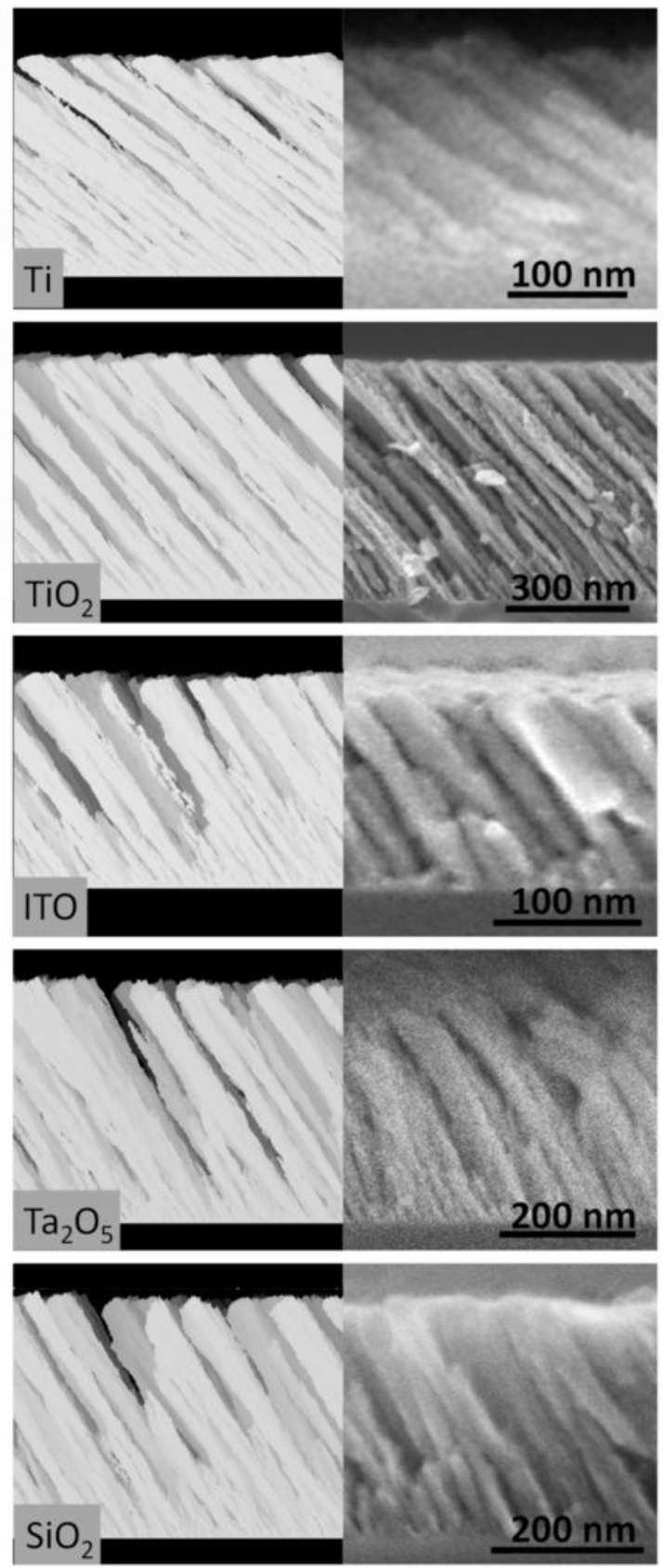